\title{
Hesperidin induces apoptosis and G0/G1 arrest in human non-small cell lung cancer A549 cells
}

\author{
RONGMU XIA, XIN SHENG, XIANLIN XU, CHUNBO YU and HONGLING LU \\ Department of Biochemistry and Molecular Biology, Zunyi Medical College, Zunyi, Guizhou 563000, P.R. China
}

Received March 27, 2017; Accepted October 27, 2017

DOI: $10.3892 /$ ijmm.2017.3250

\begin{abstract}
Lung cancer has high incidence and mortality rates worldwide. In the present study, the mechanisms by which hesperidin decreases the viability and induces the apoptosis of human non-small cell lung cancer (NSCLC) A549 cells were investigated. Initially, MTT and flow cytometric assays were performed to evaluate the effects of hesperidin on the viability and apoptosis of A549 cells and human normal lung epithelial BEAS-2B cells. The results revealed that hesperidin has no negative effects on the human normal lung epithelial BEAS-2B cells and the viability of cells treated with various concentrations of hesperidin was inhibited in a time- and dose-dependent manner compared with the control groups. Subsequently, the expression levels of proteins involved in the mitochondria-associated apoptotic pathway were studied by western blot analysis. Hesperidin was identified to induce A549 cell apoptosis by downregulating the levels of B-cell lymphoma-2 (Bcl-2) and Bcl extra large protein and simultaneously upregulating the levels of Bcl-2-associated $\mathrm{X}$ protein, BH3 interacting-domain death agonist (Bid), tBid, cleaved caspase-9, cleaved caspase-3 and cleaved poly(adenosine diphosphate ribose)polymerase. The effect of hesperidin on the cell cycle was assessed by flow cytometry. Hesperidin was observed to cause G0/G1 arrest of A549 cells by decreasing the expression of cyclin D1 and increasing the expression of p21 and p53. In summary, it was demonstrated that hesperidin induced apoptosis through the mitochondrial apoptotic pathway and induced G0/G1 arrest in human NSCLC A549 cells. Therefore, hesperidin may be developed as a potential therapeutic drug for the treatment of NSCLC.
\end{abstract}

\section{Introduction}

Lung cancer is among the most common causes of cancerassociated mortality worldwide. According to a global data

Correspondence to: Professor Hongling Lu, Department of Biochemistry and Molecular Biology, Zunyi Medical College, 1 Xuefu Road, Zunyi, Guizhou 563000, P.R. China

E-mail: 1_hongling2@163.com

Key words: hesperidin, apoptosis, cell cycle, mitochondrial apoptotic pathway, A549 cells analysis, $\sim 1.5$ million new cases are diagnosed annually, with the majority of cases categorized as non-small cell lung carcinoma (NSCLC) (1). The detection of NSCLC at an early stage presents a significant clinical challenge; in $\sim 70 \%$ of newly confirmed cases, the disease has progressed to an advanced stage, at which the patients have missed the optimal time window for surgery (2). In addition to surgery, the use of other therapeutic approaches, such as adjuvant chemotherapy and radiation therapy, has been common over the last decades (3). However, the 5-year relative survival rate of NSCLC patients remains low (4). The antineoplastic drug cisplatin (DDP) has been widely used to treat cancer; however, this drug causes severe side effects, which may include nephrotoxicity, marrow suppression and considerable gastrointestinal reactions. Furthermore, patients often experience physical and mental distress, and DDP treatment contributes so reducing their quality of life. Therefore, it is of great significance to identify effective alternatives to DDP in order to improve the outcome, prognosis and quality of life of patients with NSCLC.

Phytochemicals are derived from plants and include traditional herbal remedies that have been used for $>2,000$ years to prevent disease or promote health. A number of preclinical animal models and human epidemiological studies have demonstrated that certain phytochemicals may have an efficient preventive effect against human cancer (5). Hesperidin, a type of flavonoid, is ubiquitous in citrus species, including orange, lemon and pomelo fruits (6). Recently, the biological properties and potential therapeutic mechanisms of hesperidin have been widely tested in laboratory-based studies. Mounting evidence indicates that hesperidin may have anti-inflammatory, antioxidant, free radical-scavenging, anti-diabetic and cardioprotective effects $(7,8)$, and have proven useful in the prevention and treatment of cancer $(9,10)$. Regarding the latter, hesperidin was observed to induce paraptosis of HepG2 hepatoblastoma cells by activating the mitogen-activated protein kinase extracellular signal-regulated kinase $1 / 2$ signaling pathway, and the mitochondrial and death receptor pathways (11-13). In addition, hesperidin was able to trigger the apoptosis of NALM-6 cells (14), Ramos cells (15) and MSTO-211H cells (16) by promoting p53 accumulation, decreasing constitutive nuclear factor $-\kappa \mathrm{B}(\mathrm{NF}-\kappa \mathrm{B})$ activity and inhibiting signaling protein 1 . Hesperidin was also reported to trigger apoptosis through the extrinsic pathway (17) and induce cell cycle arrest via the endoplasmic reticulum stress pathway in HeLa cells (18). Saiprasad et al (19) observed that hesperidin initiated apoptosis and autophagy through mediating 
Aurora-A-coupled pro-survival phosphoinositide 3-kinase/Akt/ mammalian target of rapamycin signaling cascades and glycogen synthase kinase- $3 \beta$ activity to antagonize the effect of azoxymethane on colon carcinogenesis in a mouse model.

A large number of relevant studies have been published on the suppression of lung tumorigenesis by hesperidin. Kohno et al (20) reported that hesperidin reduced the expression of proliferating cell nuclear antigen to act against 4-(methylnitrosamino)1-(3-pyridyl)-1-butanone-induced pulmonary tumorigenesis in mice. Balakrishnan and Menon (21) demonstrated that hesperidin downregulated the high expression of matrix metalloproteinases (MMPs) induced by nicotine and decreased the levels of antioxidants to act against tobacco-associated disease. Kamaraj et al (22-24) reported several mechanisms for the protective effects of hesperidin against benzo(a)pyrene-induced lung carcinogenesis in mice, including an increase in the levels of antioxidants, modulation of the expression of cyclooxygenase- 2 and MMPs, recruitment of mast cells and alteration of the antioxidant and mitochondrial status, comprising major tricarboxylic acid cycle enzyme activities and electron transport chain complex activities. Birsu Cincin et al (25) confirmed that hesperidin had a greater inhibitory effect on A549 and NCI-H358 cells compared with that on MRC-5 normal lung fibroblasts, and that this effect was associated with the fibroblast growth factor and NF- $\kappa$ B signal transduction pathways.

Thus, it has been demonstrated that the antitumor effects of hesperidin represent a promising strategy for cancer therapy. The present study was performed to better understand the pharmacological effects of hesperidin on the alteration of other molecules and the cell cycle of A549 cells.

\section{Materials and methods}

Materials. Hesperidin was purchased from Santa Cruz Biotechnology, Inc. (Dallas, TX, USA). RPMI-1640 basal culture medium, Dulbecco's modified Eagle's medium (DMEM) high-glucose basal culture medium and $0.25 \%$ trypsin were purchased from HyClone (Logan, UT, USA). Fetal bovine serum (FBS) was from Gibco (Thermo Fisher Scientific, Inc., Waltham, MA, USA). Radioimmunoprecipitation assay (RIPA) lysis buffer, phenylmethanesulfonylfluoride(PMSF), broad-spectrum phosphatase inhibitor, dimethyl sulfoxide (DMSO), MTT and DDP were purchased from Solarbio (Beijing, China). The bicinchoninic acid (BCA) protein concentration detection kit was provided by CWBio (Beijing, China). Cell culture bottles and associated consumables were purchased from Corning, Inc. (Corning, NY, USA). The Annexin V-fluorescein isothiocyanate (FITC)/propidium iodide (PI) kit (cat. no. A005-3) and the cell cycle and apoptosis analysis kit (cat. no. C00150) were supplied by 7 Sea Biotech (Shanghai, China). The rabbit anti-human antibody against $\beta$-actin (cat. no. ab8226) and horseradish peroxidase (HRP)-conjugated goat anti-rabbit immunoglobulin ( $\mathrm{Ig}) \mathrm{G}$ (cat. no. ab6721) were purchased from Abcam (Cambridge, UK). Rabbit anti-human antibodies against B-cell lymphoma-2 (Bcl-2; cat. no. 4223T), Bcl-2-associated $\mathrm{X}$ protein (Bax; cat. no. 5023T), BH3 interacting-domain death agonist (Bid)/tBid (cat. no. 2002T), B-cell lymphoma extra large protein (Bcl-xL; cat. no. 2764T), cleaved caspase-3 (cat. no. 9664T), cleaved caspase-9 (cat. no. 7237T), cleaved poly(adenosine triphosphate ribose)polymerase (PARP; cat. no. 5625T), p21 (cat. no. 2947T), p53 (cat. no. 2527T) and cyclin D1 (cat. no. 2978T) were obtained from Cell Signaling Technology, Inc. (Beverly, MA, USA).

Cell lines and culture conditions. The A549 human NSCLC cell line and the BEAS-2B human normal lung epithelial cell line were purchased from the Cell Bank of the Chinese Academy of Medical Science (Shanghai, China). A549 and BEAS-2B cells were respectively cultured in RPMI-1640 and high-glucose DMEM, each supplemented with a mixture of $10 \% \mathrm{FBS}$ and $1 \%$ penicillin/streptomycin (Solarbio) at $37^{\circ} \mathrm{C}$ in a humidified atmosphere containing $5 \% \mathrm{CO}_{2}$.

Experimental groups. Hesperidin was dissolved in DMSO to produce a $25 \mathrm{mg} / \mathrm{ml}$ stock solution and stored as aliquots in tightly sealed vials at $-20^{\circ} \mathrm{C}$. Working solutions were prepared by serial dilutions of stock solution with whole culture medium. In this study, 7 experimental groups were set as follows: A control group, a $0.5 \%$ DMSO group, several hesperidin groups (50, 75, 100 and $125 \mu \mathrm{g} / \mathrm{ml}$ hesperidin); and a DDP group $(1 \mu \mathrm{g} / \mathrm{ml} \mathrm{DDP})$ as a positive control.

Cell viability assay. The effects of hesperidin on the viability of A549 and BEAS-2B cells were detected by an MTT assay. In brief, each group of A549 cells ( $1 \times 10^{4}$ cells/well) in sextuplicate wells of a 96-well plate was incubated for $24 \mathrm{~h}$, which was followed by the addition of hesperidin or DMSO to each group (as stated above) and incubation for 24, 48 and $72 \mathrm{~h}$. After removal of the liquid, $180 \mu \mathrm{l}$ whole culture medium and MTT (20 $\mu \mathrm{l} /$ well; final concentration, $5 \mathrm{mg} / \mathrm{ml}$ ) were added into each well for a 4 -h treatment. The resulting formazan was dissolved in $100 \mu \mathrm{l}$ DMSO and the absorbance as the optical density (OD) at $490 \mathrm{~nm}$ for each well was determined with an iMark $^{\mathrm{TM}}$ Microplate Reader (Bio-Rad Laboratories, Hercules, CA, USA). The rates of cell viability inhibition by hesperidin were calculated using the following formula: $\left(\mathrm{OD}_{\text {control group }}-\mathrm{OD}_{\text {experimental group }}\right) / \mathrm{OD}_{\text {control group }}$. In addition, the morphology of individual groups of cells after the 72-h treatment with hesperidin or DDP $(1 \mu \mathrm{g} / \mathrm{ml})$ was observed using a microscope (Olympus, Tokyo, Japan), and the average cell number in each field of view was counted.

Cell apoptosis assay. A549 or BEAS-2B cells ( $1 \times 10^{6}$ cells/well) were seeded into 6-well plates for $24 \mathrm{~h}$ and then treated with DMSO or hesperidin at varying concentrations (as stated above) for 24,48 or 72 h. Following treatment with $0.25 \%$ trypsin without EDTA, the supernatant and adherent cells were harvested. Cells were resuspended and washed twice with phosphate-buffered saline (PBS). After removal of the supernatant, $400 \mu \mathrm{l}$ binding buffer from the Annexin VFITC/PI kit was added to resuspend the cells. Subsequently, $5 \mu \mathrm{l}$ Annexin V-FITC was added, followed by thorough mixing and incubation for $15 \mathrm{~min}$ in the dark at ambient temperature. Thereafter, the cells were stained with $10 \mu \mathrm{l}$ PI and incubated for $5 \mathrm{~min}$ in the dark in an ice bath. Subsequently, the percentage of apoptotic cells was determined within $30 \mathrm{~min}$ by flow cytometric analysis.

Cell cycle analysis. A549 cells ( $1 \times 10^{6}$ cells/well) were seeded into 6-well plates, incubated for $24 \mathrm{~h}$ and then treated with 

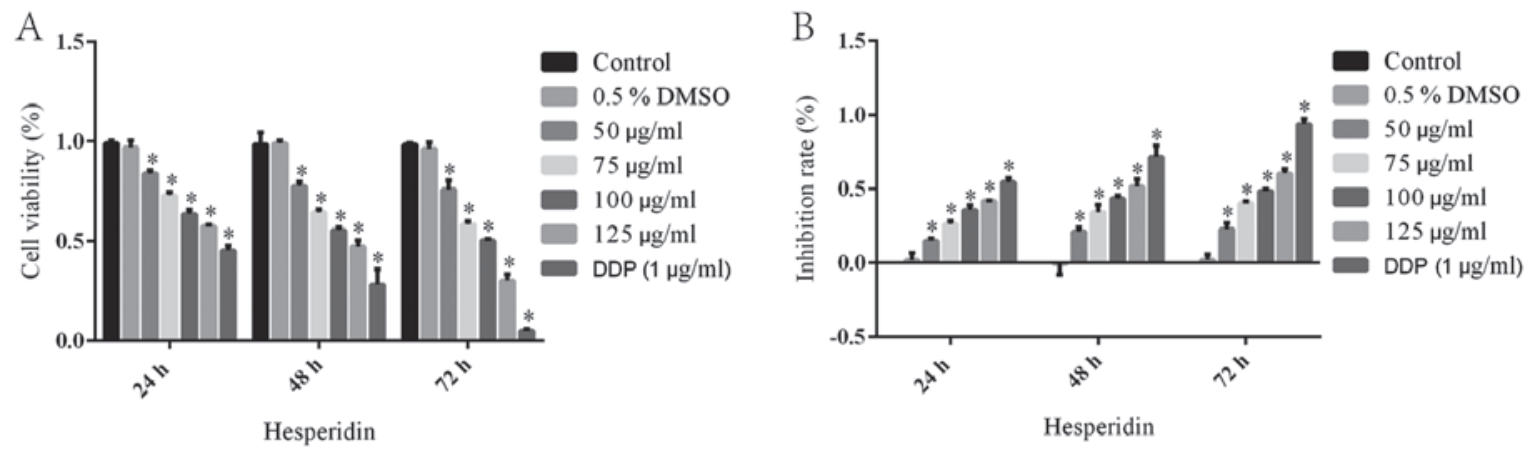

Figure 1. Effects of different concentrations of hesperidin or DDP on the viability of A549 cells. A549 cells were treated with hesperidin or DDP (1 $\mu \mathrm{g} / \mathrm{ml})$ for 24, 48 or $72 \mathrm{~h}$. (A) The relative survival rate of A549 cells was detected by an MTT assay and (B) the inhibition ratio was calculated. The survival rate of the control group was defined as $100 \%$. Values are expressed as the mean \pm standard deviation $(n=6)$. ${ }^{*} \mathrm{P}<0.05$ vs. control. DDP, cisplatin; DMSO, dimethylsulfoxide.

vehicle (DMSO) or hesperidin at varying concentrations (as stated above) for $72 \mathrm{~h}$. After treatment with $0.25 \%$ trypsin, the supernatant and detached cells were harvested. Cells were resuspended and washed twice with precooled PBS. After removal of the supernatant, $1 \mathrm{ml}$ precooled $70 \%$ ethyl alcohol was added followed by gentle mixing and incubation for $30 \mathrm{~min}$ at $4^{\circ} \mathrm{C}$ to fix the cells. After removal of the supernatant, cells were resuspended and washed with precooled PBS. Premixed PI working solution $(500 \mu \mathrm{l})$ was then added to each sample to resuspend the cells, followed by incubation for $30 \mathrm{~min}$ in the dark at $37^{\circ} \mathrm{C}$. To produce the PI working solution, $25 \mu \mathrm{l}$ PI stock solution and $20 \mu \mathrm{l}$ RNase A solution $(10 \mathrm{mg} / \mathrm{ml})$ were added to $1 \mathrm{ml}$ staining buffer and mixed gently. Within $5 \mathrm{~h}$, flow cytometric analysis of the distribution of cells in different phases of the cell cycle was performed. Red fluorescence was detected at an excitation wavelength of $488 \mathrm{~nm}$.

Western blot analysis. A549 cells ( $1 \times 10^{6}$ cells/well) were seeded in 6-well plates for $24 \mathrm{~h}$ and then treated with vehicle (DMSO) or hesperidin at varying concentrations (as stated above) for $72 \mathrm{~h}$. After removal of the supernatant, the cells in the 6-well plates were put on ice. Fifty microliters RIPA buffer, PMSF and broad-spectrum phosphatase inhibitor were added to each well. The cells in the lysis solution were filtered by centrifugation at $12,000 \mathrm{xg}$ for $15 \mathrm{~min}$ at $4^{\circ} \mathrm{C}$. The concentration of total protein was detected using the BCA protein detection kit and an iMark $^{\mathrm{TM}}$ microplate reader (Bio-Rad Laboratories, Inc.). Equal volumes of loading buffer were then added to each sample, followed by boiling for $5 \mathrm{~min}$, and the samples of denatured protein were stored at $-20^{\circ} \mathrm{C}$. Denatured protein $(40 \mu \mathrm{g} / \mathrm{lane})$ from each sample was separated by $10 \%$ sodium dodecyl sulfatepolyacrylamide gel electrophoresis (SDS-PAGE) and transferred onto a polyvinylidene fluoride membrane (cat. no. 1620177; Bio-Rad Laboratories, Inc.) by wet-transfer. The membranes were blocked for $2 \mathrm{~h}$ with $5 \%$ bovine serum albumin (cat. no. A8020; Solarbio) at ambient temperature, and then incubated with the corresponding pre-diluted rabbit anti-human primary antibodies (1:2,000 dilution) overnight at $4^{\circ} \mathrm{C}$. Anti- $\beta$-actin was used as the control. After washing in Tris-buffered saline containing Tween20 , the bound antibodies were probed with HRP-conjugated goat anti-rabbit IgG secondary antibodies (1:2,000 dilution) by incubation for $2 \mathrm{~h}$ at room temperature. Finally, the bound antibodies were visualized using an enhanced chemiluminescence (ECL) detection reagent (cat. no. 1705060; Bio-Rad Laboratories,
Inc.). The levels of each target protein relative to the control were determined by measuring the integral optical density in a ChemiDoc Touch Imaging system and quantitatively calculated using Image Laboratory software v. 5.1 (both from Bio-Rad Laboratories, Inc.).

Statistical analysis. All statistical analyses were performed using SPSS software (version 13.0; SPSS, Inc., Chicago, IL, USA). Pairwise comparisons were performed by using Student's t-test. The differences among three or more groups were determined by one-way analysis of variance followed by a Bonferroni's or Dunnett's test. Values are expressed as the mean \pm standard deviation. $\mathrm{P}<0.05$ was regarded to indicate a statistically significant difference.

\section{Results}

Effects of different concentrations of hesperidin or DDP on the viability and morphology of A549 cells. An MTT assay was performed to investigate the inhibitory effect of hesperidin on the viability of A549 cells. The cell survival rate of the control group was defined as $100 \%$ with an inhibition ratio of $0 \%$. The cell survival rate and inhibition ratio in the $0.5 \%$ DMSO group was not significantly different from that in the control group, while DDP $(1 \mu \mathrm{g} / \mathrm{ml})$ treatment caused a significant decrease in the relative cell survival rate (Fig. 1). Thus, $0.5 \%$ DMSO, as the solvent of hesperidin, had no effect on the viability of A549 cells. In addition, it was observed that the cell viability in the groups treated with hesperidin for 24,48 or $72 \mathrm{~h}$ was significantly decreased in a time- and dose-dependent manner (Fig. 1). After treatment with 50,75, 100 and $125 \mu \mathrm{g} / \mathrm{ml}$ hesperidin for $72 \mathrm{~h}$, the morphology of A549 cells was altered and the majority of the cells treated with DDP $(1 \mu \mathrm{g} / \mathrm{ml})$ were apoptotic compared with that in the control group (Fig. 2A). It was noted that the cells lost their adherence, appeared shrunken and acquired a rounded shape. In addition, the cell density per field of view was significantly decreased compared with that of the control group $(\mathrm{P}<0.05)$. However, the group treated with 0.5\% DMSO was not significantly changed ( $\mathrm{P}>0.05)$ (Fig. $2 \mathrm{~B})$. Thus, it was demonstrated that the viability of A549 cells was decreased by hesperidin, which resulted in an alteration of cell morphology.

Effect of different concentrations of hesperidin and DDP on the induction of apoptosis in A549 cells. To explore the molecular 

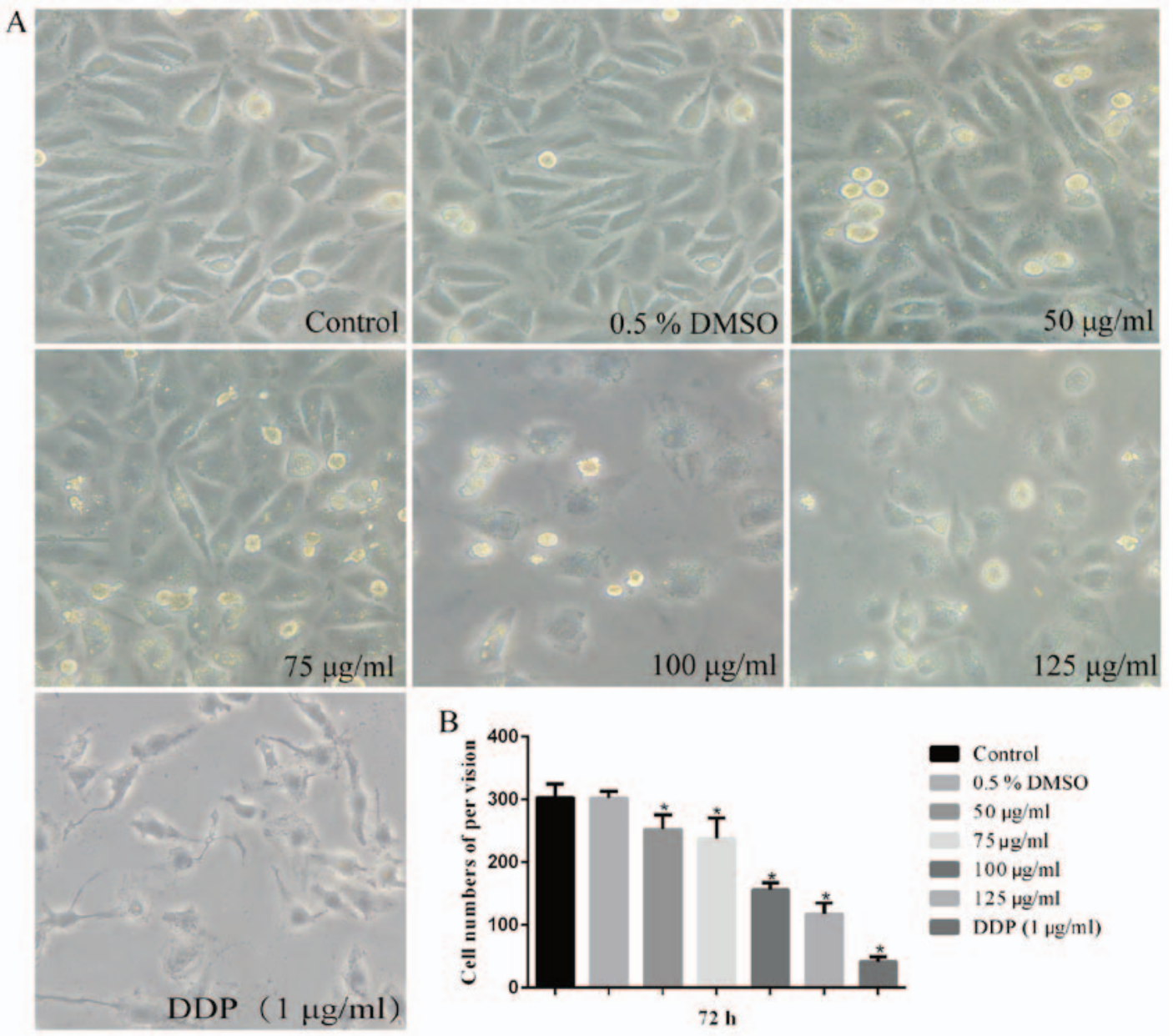

Figure 2. Effects of different concentrations of hesperidin or DDP on the morphology of A549 cells. A549 cells were cultured with hesperidin or DDP ( $1 \mu \mathrm{g} / \mathrm{ml})$ for $72 \mathrm{~h}$. (A) The morphological changes of A549 cells were observed using microscopy (magnification, x400). (B) The cell numbers in ten randomly selected fields of view were counted and averaged. Values are expressed as the mean \pm standard deviation. ${ }^{*} \mathrm{P}<0.05$ vs. control. DDP, cisplatin; DMSO, dimethylsulfoxide.

mechanisms by which hesperidin reduced the viability of A549 cells, a flow cytometric apoptosis assay was performed. Cells were treated for 24,48 or $72 \mathrm{~h}$ with different concentrations of hesperidin and stained with Annexin V-FITC/PI. The results indicated that the apoptotic rate in the control group was $<5 \%$, and the necrotic rate in all groups was $<2 \%$, which was considered acceptable (Fig. 3A-C). The apoptotic rate in the $0.5 \%$ DMSO group was not significantly different compared with that in the control group (Fig. 3), indicating that $0.5 \%$ DMSO (vehicle) had no effect on the survival rate of A549 cells. The apoptotic rates in the groups treated with different concentrations of hesperidin for different durations were increased compared with those in the control group, and this effect was identified to be time- and dose-dependent (Fig. 3D).

Furthermore, A549 cells were treated with different concentrations of hesperidin for $72 \mathrm{~h}$. The results confirmed that hesperidin elevated the expression levels of mitochondrial apoptotic pathway-associated proteins (Bax, Bid, tBid, cleaved caspase-3, cleaved caspase- 9 and cleaved PARP), while significantly reducing the expression levels of Bcl-2 and Bcl-xL compared with the control in a concentration-dependent manner $(\mathrm{P}<0.05)$ (Fig. 4). The relative expression levels of mitochondrial apoptotic pathway-associated proteins in the $0.5 \%$ DMSO group were not significantly different compared with those in the control group ( $\mathrm{P}>0.05)$ (Fig. 4B), indicating that $0.5 \%$ DMSO had no effect on these proteins in A549 cells. In conclusion, it was demonstrated that hesperidin induced apoptosis in A549 cells, which was regulated by the expression of mitochondrial apoptotic pathway-associated proteins.

Effects of different concentrations of hesperidin or DDP on the viability and apoptosis of BEAS-2B normal human lung epithelial cells. BEAS-2B cells were treated with hesperidin or DDP $(1 \mu \mathrm{g} / \mathrm{ml})$ as described above. The results of the MTT and flow cytometry assays demonstrated that there were no significant differences between the hesperidin- and $0.5 \%$ DMSO-treated cells and the control group ( $\mathrm{P}>0.05)$ (Fig. 5A), whereas DDP-treated $(1 \mu \mathrm{g} / \mathrm{ml})$ cells exhibited significantly decreased viability $(\mathrm{P}<0.05)$ (Fig. $5 \mathrm{~A}-\mathrm{a})$, as well as notably increased rates of inhibition of proliferation $(\mathrm{P}<0.05$ (Fig. $5 \mathrm{~A}-\mathrm{b})$ and apoptosis $(\mathrm{P}<0.05)$ (Fig. 5B).

Hesperidin causes G0/G1 phase arrest of A549 cells. The effect of hesperidin on the cell cycle distribution of A549 cells was detected by flow cytometry (Fig. 6A). The G0/G1 phase population in the $0.5 \%$ DMSO group was not significantly different from that in the control group ( $\mathrm{P}>0.05)$ (Fig. 6B). However, the proportion of cells in G0/G1 phase in the groups treated with various concentrations of hesperidin for different 

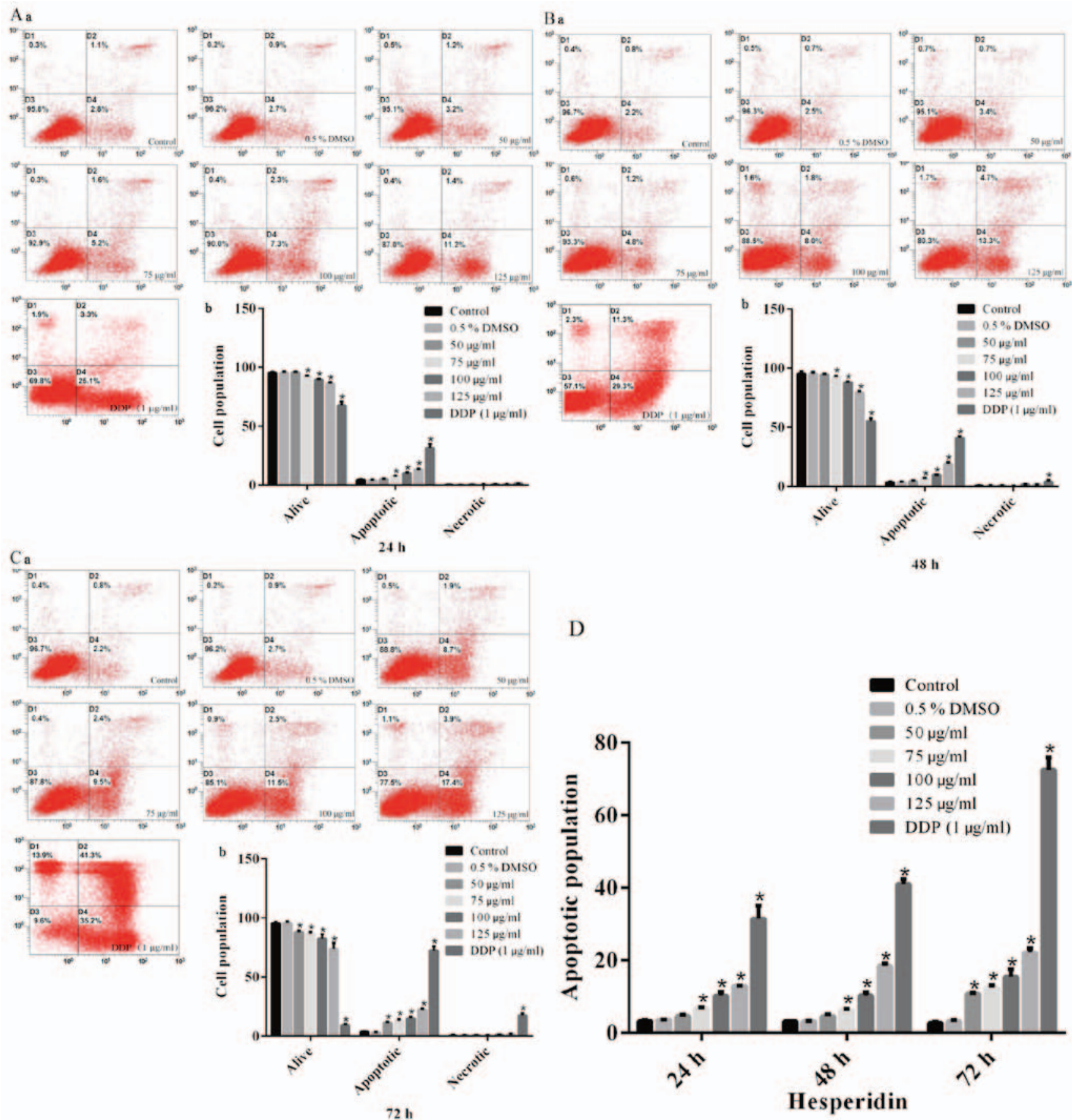

$48 \mathrm{~h}$

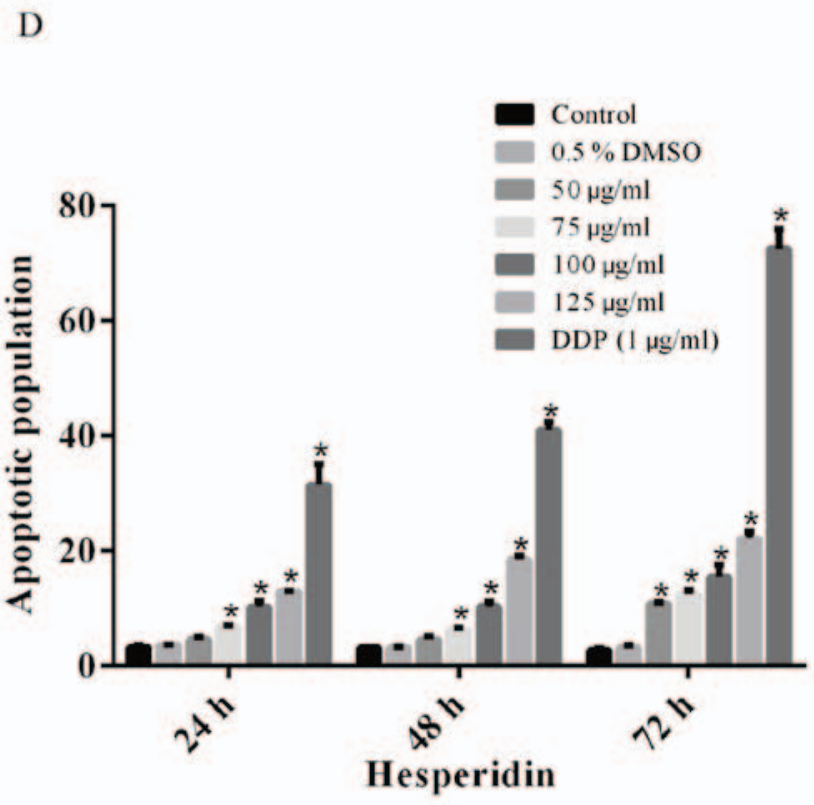

Figure 3. Hesperidin or DDP treatment induce apoptosis in A549 cells. (A-a) Flow cytometry dot plots at 24 h; (A-b) quantitative analysis of viable, apoptotic and necrotic A549 cells. (B and C) Analysis of apoptosis at (B) $48 \mathrm{~h}$ and (C) $72 \mathrm{~h}$. (D) Results of a quantitative analysis of apoptotic A549 cells treated with different concentrations of hesperidin for different durations. Values are expressed as the mean \pm standard deviation. ${ }^{*}<0.05$ vs. control. DDP, cisplatin; DMSO, dimethylsulfoxide.

durations were significantly increased compared with those in the control group, indicating cell-cycle/growth arrest in G0/G1 phase $(\mathrm{P}<0.05)$ (Fig. 6B).

A549 cells were treated with different concentrations of hesperidin for $72 \mathrm{~h}$ and the expression of various cell cycleassociated proteins was assessed by western blot analysis. The results confirmed that hesperidin elevated the relative expression levels of p21 and p53, while reducing the expression levels of the cell cycle-regulatory protein cyclin D1 (Fig. 7A). Quantitative analysis revealed significant differences compared with the control $(\mathrm{P}<0.05)$ (Fig. 7B). The 0.5\% DMSO group exhibited no significant differences compared with the control group ( $\mathrm{P}>0.05)$ (Fig. 7). These results demonstrated that hesperidin induced $\mathrm{G} 0 / \mathrm{G} 1$ phase arrest by regulating the relative expression of cell cycle-associated proteins in A549 cells.

\section{Discussion}

The present study indicated a significant inhibitory effect of hesperidin on the viability and cell cycle of A549 NSCLC cells. The cell viability was inhibited by hesperidin through the mitochondrial apoptotic pathway as well as induction of G0/G1 


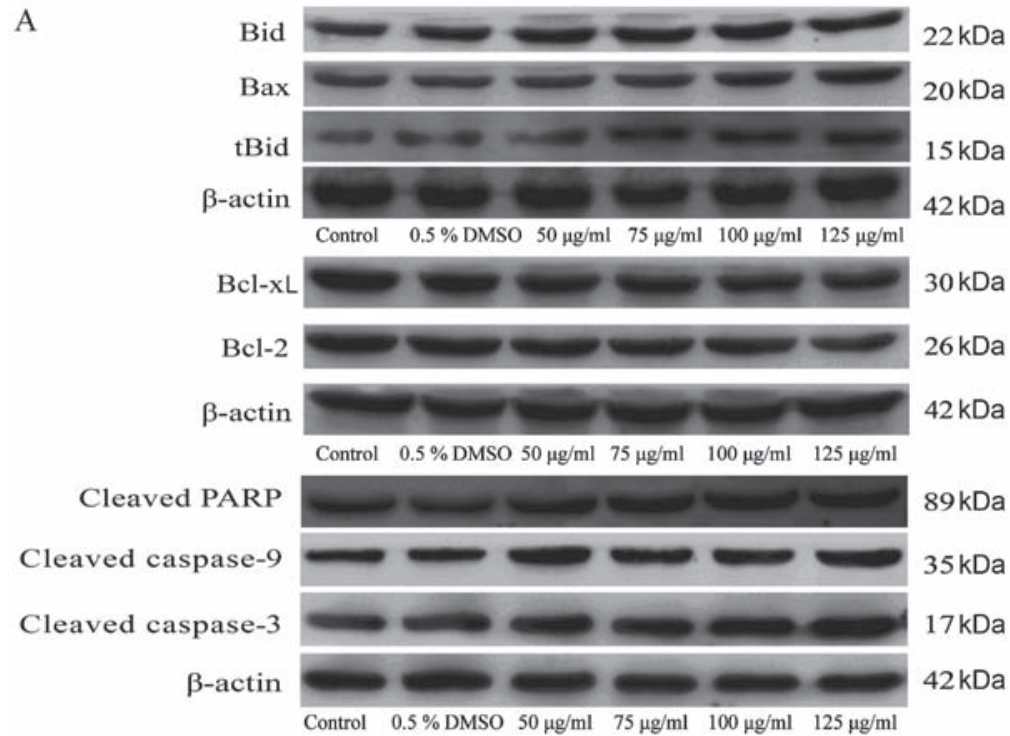

B

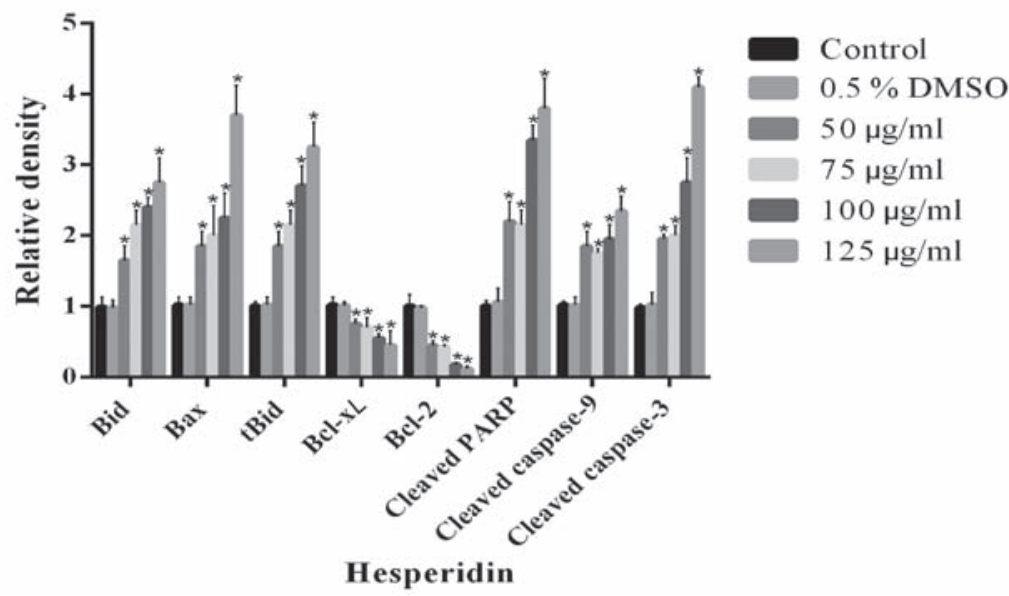

Figure 4. Expression of mitochondrial apoptotic pathway-associated proteins in A549 cells treated with hesperidin. A549 cells were cultured with hesperidin for $72 \mathrm{~h}$ and the protein expression was measured by western blot analysis. (A) Images of western blot analyses; (B) quantitative analysis of the relative expression levels of the target proteins. Values are expressed as the mean \pm standard deviation. ${ }^{*} \mathrm{P}<0.05$ vs. control. DDP, cisplatin; DMSO, dimethylsulfoxide; Bcl-2, B-cell lymphoma-2; Bcl-xL, Bcl extra large protein; Bax, Bcl-2-associated X protein; Bid, BH3 interacting-domain death agonist; PARP, poly(adenosine diphosphate ribose)polymerase.

arrest, accompanied by changes in cell morphology, in a timeand dose-dependent manner, while not exerting any negative effects on BEAS-2B human normal lung epithelial cells.

Tumor occurrence and development are associated not only with dedifferentiation and excessive multiplication of tumor cells, but are also with the suppression of apoptosis. To investigate the mechanisms underlying the effect of hesperidin on apoptosis, apoptosis-associated signaling molecules were detected in A549 cells, which demonstrated that cell apoptosis was increased after treatment with hesperidin. Cell apoptosis is regulated by multiple apoptosis-promoting proteins, such as Bax, Bad and Bid, and is upregulated by a family of apoptosis-inhibiting proteins, which include Bcl-2 and Bcl-xL. The mitochondrial apoptotic pathway has a critical role in cell apoptosis. After cytochrome $\mathrm{c}$ is released from the mitochondria into the cytosol, cell apoptosis is promoted through the activation of caspase-9 and subsequent downstream factors, such as caspase- 3 and cleaved PARP (26). The balance between apoptosis-promoting and apoptosis-inhibiting proteins is crucial for the process of apoptosis $(26,27)$. The results of the present study suggested that the treatment of A549 cells with hesperidin upregulated the relative levels of mitochondrial apoptotic pathway-associated proteins, including Bax, Bid, tBid, cleaved caspase- 9 and cleaved caspase-3, while downregulating the relative expression levels of $\mathrm{Bcl}-2$ and $\mathrm{Bcl}-\mathrm{xL}$. Furthermore, an increase in cleaved PARP, which is one of the downstream products of caspase-3, was observed. These results are in line with those of a previous study (25). Therefore, it was indicated that A549 cell apoptosis is induced by hesperidin via activation of the mitochondrial apoptotic pathway.

Furthermore, cell cycle arrest is associated with the triggering of cell apoptosis and usually occurs during the induction of tumor cell apoptosis (28). It was observed that A549 cells underwent cell cycle arrest in G0/G1 phase after treatment with hesperidin. Cell cycle arrest has also been linked to the inhibition of the proliferation of cancer cells (29). The cell cycle is positively regulated by certain cell cycle-associated proteins and negatively regulated by certain inhibitory factors of cyclin-dependent kinases (CDKs), such as p21, p16 and p27. In the present study, it was indicated that the expression of $\mathrm{p} 21$ 

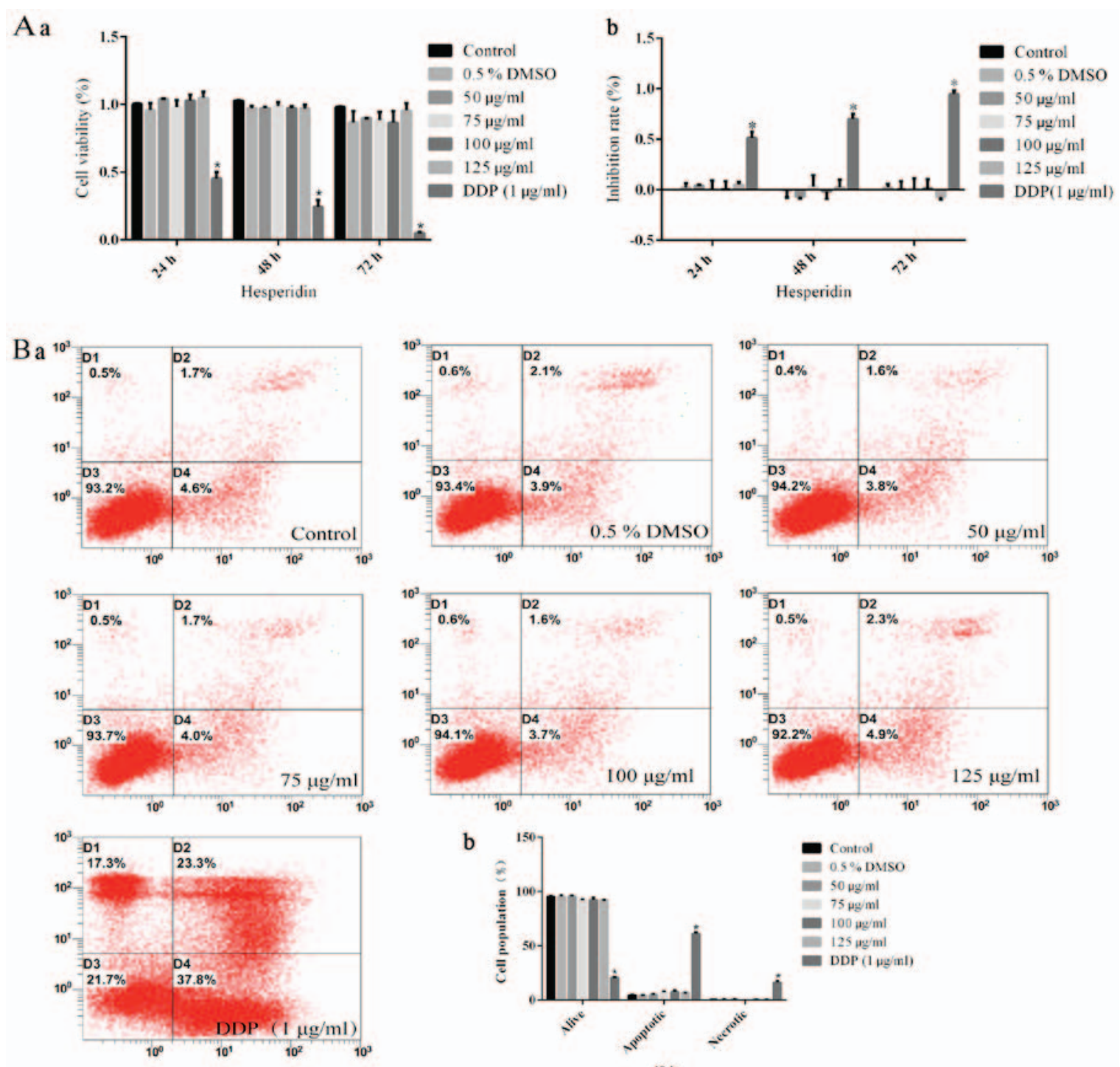

b

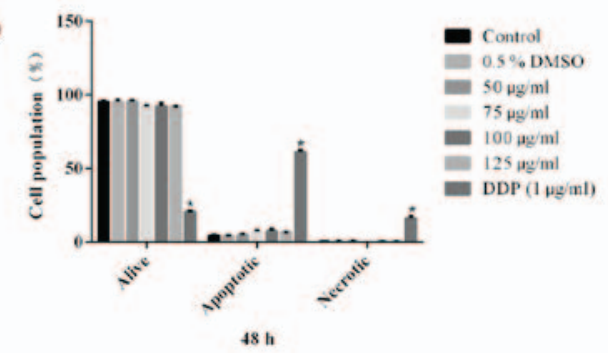

Figure 5. Effect of hesperidin or DDP on the viability and apoptosis of human normal lung epithelial BEAS-2B cells. (A-a) Cell viability and (A-b) inhibition ratio of BEAS-2B cells as measured by an MTT assay. (B-a) Apoptosis of BEAS-2B cells as determined by flow cytometry and (B-b) quantified proportions of viable, apoptotic and necrotic cells. Values are expressed as the mean \pm standard deviation. ${ }^{\text {P }}<0.05$ vs. control. DDP, cisplatin; DMSO, dimethylsulfoxide.

was upregulated, while cyclin D1 was downregulated after the treatment of A549 cells with hesperidin in a concentrationdependent manner. Cyclin D1 is the most important protein in the regulation of the $\mathrm{G} 1$ phase, and its expression is altered during tumorigenesis (30). CDKs regulate the cell cycle at different levels by affecting the assembly of cyclins and CDK subunits. The G1/S phase transition is negatively regulated by $\mathrm{p} 21$ and $\mathrm{p} 27$, which is achieved through degrading certain cyclin/CDK complexes (31). In a previous study, hesperidin was able to inhibit the G1/S transition by decreasing the relative expression levels of cyclin D1, while increasing p21 levels, which was associated with G0/G1 phase arrest (32). In addition, after treatment with hesperidin, elevated expression of p53 was observed in A549 cells, which is also associated with cell cycle arrest and apoptosis (33-35).

Considering that p53 is an important factor regulating the expression of $\mathrm{p} 21$, it was hypothesized that hesperidin induced G0/G1 arrest of A549 cells by upregulating the levels of p53, thereby upregulating the levels of p21 and in turn inhibiting the conjugation of cyclin D1/CDK complexes. To the best of our knowledge, the present study was the first to report on the inhibitory effects of hesperidin on the cell cycle of A549 cells. However, further study is necessary to elucidate the underlying mechanisms.

Taken together, the results of the present study demonstrated that hesperidin induces apoptosis in A549 cells by activating the mitochondrial apoptotic pathway, and inhibits the proliferation of A549 cells through induction of G0/G1 phase arrest by significantly upregulating the expression of p21 and p53, as well as downregulating cyclin D1. Hence, it was indicated that hesperidin may be developed as a potential therapeutic drug against NSCLC in the future.

\section{Acknowledgements}

The authors would like to thank Dr Bo Zhang (Department of Biochemistry and Molecular Biology, Zunyi Medical College, Zunyi) and Dr Fei He (Translation Medicine Collaborative Innovation Center of Shenzhen People's Hospital, Shenzhen) for their efforts in modifying the manuscript and Dr Kuifeng 

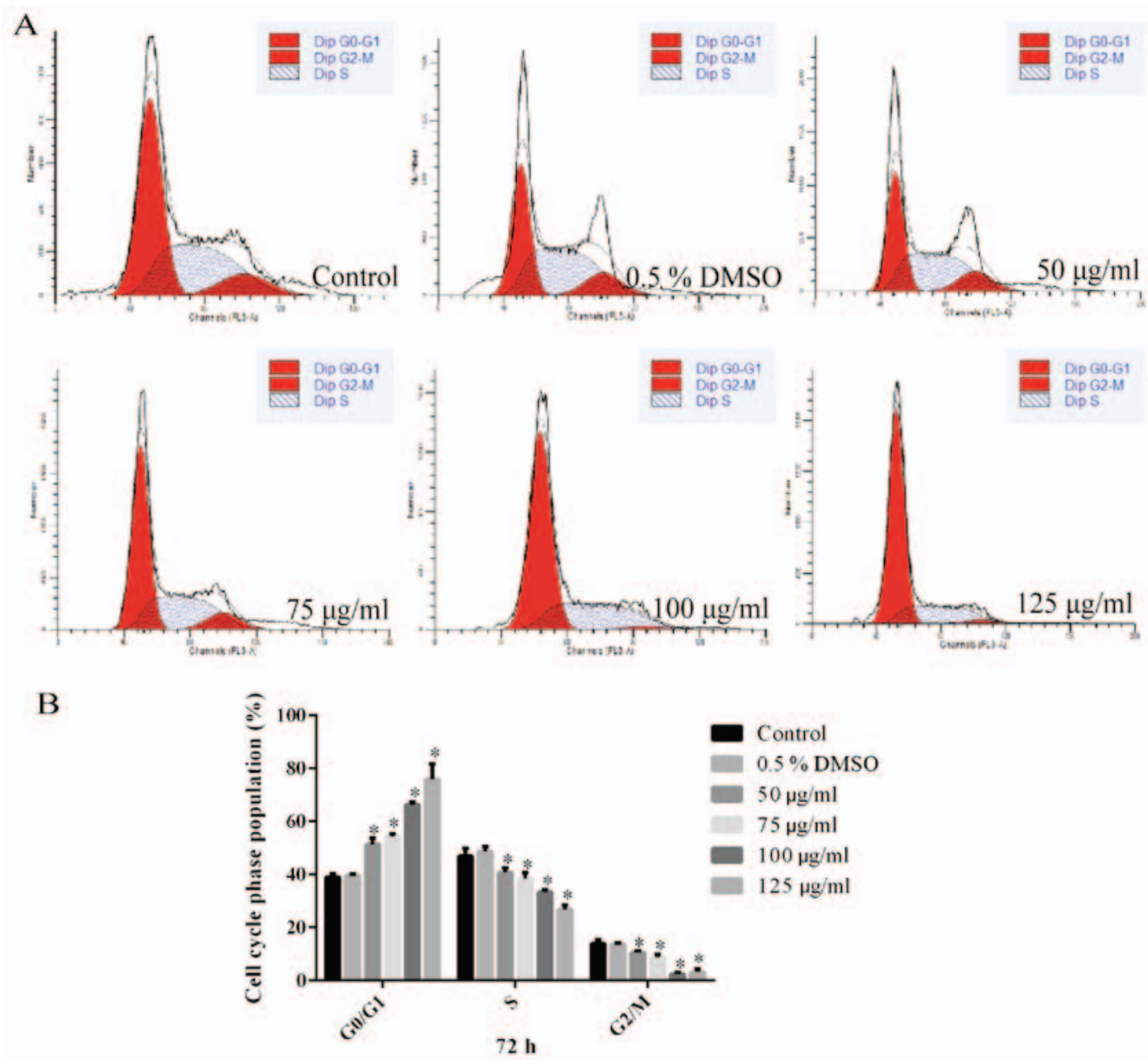

Figure 6. Hesperidin induces G0/G1 phase arrest of A549 cells. A549 cells were treated with hesperidin for $72 \mathrm{~h}$. The cell cycle distribution was detected by flow cytometry. (A) Flow cytometric cell cycle distribution; (B) quantitative analysis of cells in different phases of the cell cycle. Values are expressed as the mean \pm standard deviation. "P $<0.05$ vs. control. DDP, cisplatin; DMSO, dimethylsulfoxide.

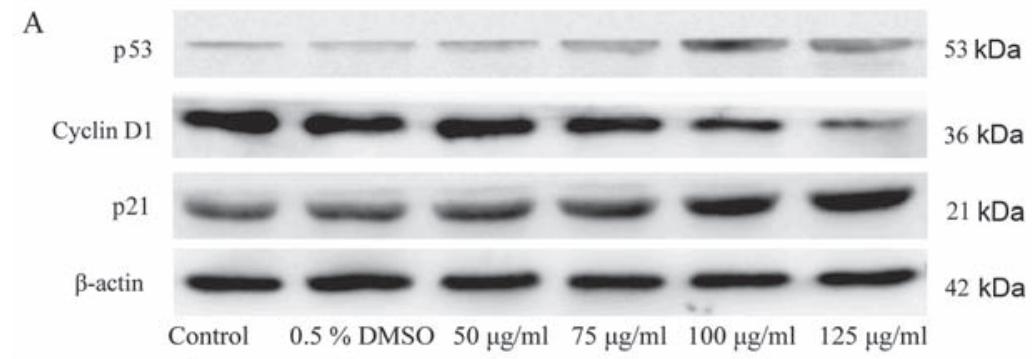

$\mathrm{B}$

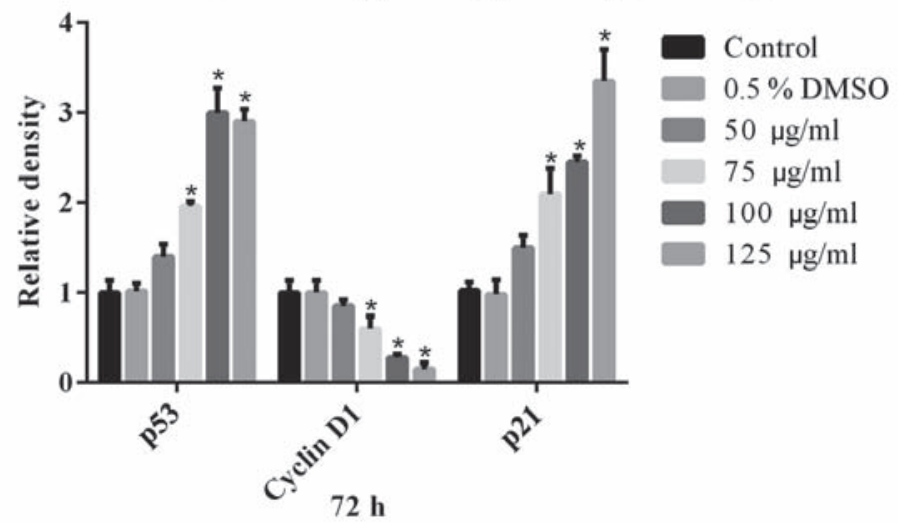

Figure 7. Expression of cell cycle-associated proteins after treatment of A549 cells with hesperidin for $72 \mathrm{~h}$. Western blot analysis was used to evaluate protein expression. (A) Representative western blot images and (B) quantitative analysis of the relative expression levels of the target proteins. Values are expressed as the mean \pm standard deviation. ${ }^{*} \mathrm{P}<0.05$ vs. control. DDP, cisplatin; DMSO, dimethylsulfoxide. 
Wang (Qinhao Biotechnology Co., Ltd., Shanghai) for his technical assistance. This study was financed by the Department of Education of Guizhou Province [grant no. (2015)375] and the Grant of Key Subject Construction of Zunyi Medical College (grant no. SSDJS201623).

\section{References}

1. NSCLC Meta-analysis Collaborative Group: Preoperative chemotherapy for non-small-cell lung cancer: A systematic review and meta-analysis of individual participant data. Lancet 383: 1561-1571, 2014

2. Saintigny $P$ and Burger JA: Recent advances in non-small cell lung cancer biology and clinical management. Discov Med 13: 287-297, 2012.

3. He J, Shen J, Yang C, Jiang L, Liang W, Shi X, Xu X and He J: Adjuvant chemotherapy for the completely resected stage IB nonsmall cell lung cancer: A systematic review and meta-analysis Medicine (Baltimore) 94: e903, 2015.

4. DeSantis CE, Lin CC, Mariotto AB, Siegel RL, Stein KD, Kramer JL, Alteri R, Robbins AS and Jemal A: Cancer treatment and survivorship statistics, 2014. CA Cancer J Clin 64: 252-271, 2014.

5. Singh BN, Singh HB, Singh A, Naqvi AH and Singh BR: Dietary phytochemicals alter epigenetic events and signaling pathways for inhibition of metastasis cascade: Phytoblockers of metastasis cascade. Cancer Metastasis Rev 33: 41-85, 2014.

6. Lu Y, Zhang C, Bucheli P and Wei D: Citrus flavonoids in fruit and traditional Chinese medicinal food ingredients in China. Plant Foods Hum Nutr 61: 57-65, 2006.

7. Roohbakhsh A, Parhiz H, Soltani F, Rezaee R and Iranshahi M: Molecular mechanisms behind the biological effects of hesperidin and hesperetin for the prevention of cancer and cardiovascular diseases. Life Sci 124: 64-74, 2015.

8. Knekt P, Kumpulainen J, Järvinen R, Rissanen H, Heliövaara M, Reunanen A, Hakulinen T and Aromaa A: Flavonoid intake and risk of chronic diseases. Am J Clin Nutr 76: 560-568, 2002.

9. Ahmadi A, Shadboorestan A, Nabavi SF, Setzer WN and Nabavi SM: The role of hesperidin in cell signal transduction pathway for the prevention or treatment of cancer. Curr Med Chem 22: 3462-3471, 2015.

10. Tanaka T, Tanaka T, Tanaka M and Kuno T: Cancer chemoprevention by citrus pulp and juices containing high amounts of $\beta$-cryptoxanthin and hesperidin. J Biomed Biotechnol 2012: 516981, 2012.

11. Yumnam S, Hong GE, Raha S, Saralamma VV, Lee HJ, Lee WS, Kim EH and Kim GS: Mitochondrial dysfunction and $\mathrm{Ca}(2+)$ overload contributes to hesperidin induced paraptosis in hepatoblastoma cells, HepG2. J Cell Physiol 231: 1261-1268, 2016.

12. Banjerdpongchai R, Wudtiwai B, Khaw-On P, Rachakhom W, Duangnil N and Kongtawelert P: Hesperidin from Citrus seed induces human hepatocellular carcinoma HepG2 cell apoptosis via both mitochondrial and death receptor pathways. Tumour Biol 37: 227-237, 2016.

13. Yumnam S, Park HS, Kim MK, Nagappan A, Hong GE, Lee HJ, Lee WS, Kim EH, Cho JH, Shin SC, et al: Hesperidin induces paraptosis like cell death in hepatoblastoma, HepG2 cells: Involvement of ERK1/2 MAPK [corrected]. PLoS One 9: e101321, 2014

14. Ghorbani A, Nazari M, Jeddi-Tehrani M and Zand H: The citrus flavonoid hesperidin induces $\mathrm{p} 53$ and inhibits NF- $\kappa \mathrm{B}$ activation in order to trigger apoptosis in NALM-6 cells: Involvement of PPAR $\gamma$-dependent mechanism. Eur J Nutr 51: 39-46, 2012.

15. Nazari M, Ghorbani A, Hekmat-Doost A, Jeddi-Tehrani M and Zand $\mathrm{H}$ : Inactivation of nuclear factor- $\mathrm{KB}$ by citrus flavanone hesperidin contributes to apoptosis and chemo-sensitizing effect in Ramos cells. Eur J Pharmacol 650: 526-533, 2011.

16. Lee KA, Lee SH, Lee YJ, Baeg SM and Shim JH: Hesperidin induces apoptosis by inhibiting Sp1 and its regulatory protein in MSTO-211H cells. Biomol Ther (Seoul) 20: 273-279, 2012.

17. Bartoszewski R, Hering A, Marszałł M, Stefanowicz Hajduk J, Bartoszewska S, Kapoor N, Kochan K and Ochocka R: Mangiferin has an additive effect on the apoptotic properties of hesperidin in Cyclopia sp. tea extracts. PLoS One 9: e92128, 2014.
18. Wang Y, Yu H, Zhang J, Gao J, Ge X and Lou G: Hesperidin inhibits HeLa cell proliferation through apoptosis mediated by endoplasmic reticulum stress pathways and cell cycle arrest. BMC Cancer 15: 682, 2015.

19. Saiprasad G, Chitra P, Manikandan R and Sudhandiran G: Hesperidin induces apoptosis and triggers autophagic markers through inhibition of Aurora-A mediated phosphoinositide-3kinase/Akt/mammalian target of rapamycin and glycogen synthase kinase-3 beta signalling cascades in experimental colon carcinogenesis. Eur J Cancer 50: 2489-2507, 2014.

20. Kohno H, Taima M, Sumida T, Azuma Y, Ogawa H and Tanaka T: Inhibitory effect of mandarin juice rich in beta-cryptoxanthin and hesperidin on 4-(methylnitrosamino)-1-(3-pyridyl)-1butanone-induced pulmonary tumorigenesis in mice. Cancer Lett 174: 141-150, 2001.

21. Balakrishnan A and Menon VP: Effect of hesperidin on matrix metalloproteinases and antioxidant status during nicotineinduced toxicity. Toxicology 238: 90-98, 2007.

22. Kamaraj S, Anandakumar P, Jagan S, Ramakrishnan G and Devaki T: Hesperidin attenuates mitochondrial dysfunction during benzo(a)pyrene-induced lung carcinogenesis in mice. Fundam Clin Pharmacol 25: 91-98, 2011.

23. Kamaraj S, Anandakumar P, Jagan S, Ramakrishnan G and Devaki T: Modulatory effect of hesperidin on benzo(a)pyrene induced experimental lung carcinogenesis with reference to COX-2, MMP-2 and MMP-9. Eur J Pharmacol 649: 320-327, 2010.

24. Kamaraj S, Ramakrishnan G, Anandakumar P, Jagan S and Devaki T: Antioxidant and anticancer efficacy of hesperidin in benzo(a)pyrene induced lung carcinogenesis in mice. Invest New Drugs 27: 214-222, 2009.

25. Birsu Cincin Z, Unlu M, Kiran B, Sinem Bireller E, Baran Y and Cakmakoglu B: Anti-proliferative, apoptotic and signal transduction effects of hesperidin in non-small cell lung cancer cells. Cell Oncol (Dordr) 38: 195-204, 2015.

26. Eichhorn JM, Alford SE, Sakurikar N and Chambers TC: Molecular analysis of functional redundancy among ant-apoptotic Bcl-2 proteins and its role in cancer cell survival. Exp Cell Res 322: 415-424, 2014

27. Silva RD, Manon S, Gonçalves J, Saraiva L and Côrte-Real M: The importance of humanized yeast to better understand the role of bcl-2 family in apoptosis: Finding of novel therapeutic opportunities. Curr Pharm Des 17: 246-255, 2011.

28. Dai HY, Liu L, Qin SK, He XM and Li SY: Lobaplatin suppresses proliferation and induces apoptosis in the human colorectal carcinoma cell Line LOVO in vitro. Biomed Pharmacother 65: 137-141, 2011.

29. Wu Z, Liu B, e C, Liu J, Zhang Q, Liu J, Chen N, Chen R and Zhu R: Resveratrol inhibits the proliferation of human melanoma cells by inducing G1/S cell cycle arrest and apoptosis. Mol Med Rep 11: 400-404, 2015.

30. Kim JK and Diehl JA: Nuclear cyclin D1: An oncogenic driver in human cancer. J Cell Physiol 220: 292-296, 2009.

31. Orlando S, Gallastegui E, Besson A, Abril G, Aligué R, Pujol MJ and Bachs O: p27Kip1 and p21Cip1 collaborate in the regulation of transcription by recruiting cyclin-Cdk complexes on the promoters of target genes. Nucleic Acids Res 43: 6860-6873, 2015.

32. Shaltiel IA, Krenning L, Bruinsma W and Medema RH: The same, only different - DNA damage checkpoints and their reversal throughout the cell cycle. J Cell Sci 128: 607-620, 2015.

33. Li L, Dai HJ, Ye M, Wang SL, Xiao XJ, Zheng J, Chen HY, Luo YH and Liu J: Lycorine induces cell-cycle arrest in the G0/G1 phase in K562 cells via HDAC inhibition. Cancer Cell Int 12: 49, 2012.

34. Kaplon J, van Dam L and Peeper D: Two-way communication between the metabolic and cell cycle machineries: The molecular basis. Cell Cycle 14: 2022-2032, 2015.

35. Wang $\mathrm{H}$, Zhang $\mathrm{X}$, Teng $\mathrm{L}$ and Legerski RJ: DNA damage checkpoint recovery and cancer development. Exp Cell Res 334: 350-358, 2015. 